\title{
Bronchiectasis in patients with chronic obstructive pulmonary disease
}

\author{
Milesh Jung Sijapati ${ }^{1}$, Narayan Bikram Thapa ${ }^{2}$, Rajendra Rijal ${ }^{1}$, Shiva \\ Raj KC ${ }^{3}$, Poojyashree Karki ${ }^{4}$ \\ ${ }^{I}$ Department of Internal Medicine, KIST Medical College \& Teaching Hospital, Lalitpur, Nepal \\ ${ }^{2}$ Department of Imaging \& Radio diagnosis, KIST Medical College \& Teaching Hospital, Lalitpur, Nepal \\ ${ }^{3}$ Department of Pathology, KIST Medical College \& Teaching Hospital, Lalitpur, Nepal \\ ${ }^{4}$ Department of Ophthalmology, KIST Medical College \& Teaching Hospital, Lalitpur, Nepal
}

\author{
Keywords: \\ Bronchiectasis; \\ COPD; \\ Exacerbation; \\ Klebsiella Pneumoniae; \\ Obstructive; \\ Pseudomonas Aeruginosa;
}

\begin{abstract}
Background: Chronic obstructive pulmonary disease is most common disease entity and third leading cause of mortality worldwide. The presence of bronchiectasis in severe chronic obstructive pulmonary disease patients had led to frequent exacerbation requiring hospitalizations. The purpose of this study was to identify the factors associated with bronchiectasis, using routine data collected during medical visits from patients diagnosed with chronic obstructive pulmonary disease.

Materials and Methods: This is hospital based cross sectional study conducted on 120 chronic obstructive pulmonary disease patients. All patients were subjected through history, examination, pulmonary function test, sputum samples and imaging of chest.

Results: Out of 120 patients among them 67 patients (55.8\%) were chronic obstructive pulmonary disease without bronchiectasis while 53 patients $(44.1 \%$ ) had chronic obstructive pulmonary disease with bronchiectasis. Thirty patients $(56.6 \%)$ having chronic obstructive pulmonary disease with bronchiectasis and 18 patient $(26.8 \%)$ having chronic obstructive pulmonary disease without bronchiectasis had exacerbation in one year requiring hospitalisation. Most common organisms isolated were Pseudomonas Aeruginosa in 20 patients $(55.5 \%)$, Klebsillae Pneumoniae in 6 patients (16.6\%) among chronic obstructive pulmonary disease with bronchiectasis group.

Conclusion: Chronic obstructive pulmonary disease patient with severe airflow limitation, with frequent exacerbation and colonisation with isolation of organisms resulting in bronchiectasis requiring frequent hospitalisation.
\end{abstract}

\section{Correspondence:}

Dr. Milesh Jung Sijapati, MD

Associate Professor, Department of Internal Medicine,

KIST Medical College \& Teaching Hospital, Imadole, Lalitpur, Nepal ORCID ID: 0000-0001-6797-4764

Email: drmileshjung@yahoo.com

Reveived : April $7^{\text {th }}, 2018$; Accepted : July $5^{\text {th }}, 2018$; Published : September $1^{\text {st }}, 2018$

Citation: Sijapati MJ, Thapa NB, Rijal R, KC Shiva R, Karki P. Bronchiectasis in patients with chronic obstructive pulmonary disease. J Pathol Nep 2018;8:1346-9. DOI: 10.3126/jpn.v8i2.20870

Copyright: This is an open-access article distributed under the terms of the Creative Commons Attribution 4.0 International License, which permits unrestricted use, distribution, and reproduction in any medium, provided the original author and source are credited.

\section{INTRODUCTION}

Bronchiectasis and chronic Obstructive Pulmonary Disease (COPD) share many characteristics, from both the physiopathology ${ }^{1,2}$ and clinical-functional viewpoints. ${ }^{3,4}$ The relationship between them can be summarized in two observations. First, they are both common conditions; especially in older patients it is, therefore, unsurprising to see patients with simultaneous COPD and bronchiectasis. Second, some authors have observed an association between the two, reporting the presence of bronchiectasis in up to $50 \%$ of patients with moderate to severe COPD; this suggests that there may be a causal relationship in which COPD is a 
Table 1: Baseline characteristics of patients

\begin{tabular}{|c|c|c|c|}
\hline & COPD without bronchiectasis & COPD with bronchiectasis & P-value \\
\hline Number & $67 \mathrm{pts}(55.8 \%)$ & $53 \mathrm{pts}(44.1 \%)$ & 0.02 \\
\hline Age & $60 \pm 10$ & $53 \pm 12$ & 0.05 \\
\hline Male & $37(55.2 \%)$ & $40(75.4 \%)$ & 0.02 \\
\hline Female & $30(44.7 \%)$ & $13(24.5 \%)$ & 0.02 \\
\hline Packed year smoked & $35 \pm 6.5$ & $30 \pm 10.2$ & 0.02 \\
\hline Daily breathlessness & $30(44.7 \%)$ & $20(37.7 \%)$ & 0.03 \\
\hline \multicolumn{4}{|l|}{ Cough } \\
\hline Mucoid & $30(52.6 \%)$ & $23(43.3 \%)$ & 0.02 \\
\hline Mucopurulent & $27(40.2 \%)$ & $20(37.7 \%)$ & 0.02 \\
\hline Purulent & $10(14.9 \%)$ & $10(18.8 \%)$ & 0.02 \\
\hline FEV1 & $53 \pm 4.50$ & $42 \pm 4.28$ & 0.03 \\
\hline FEV1/FVC & $62 \pm 5.69$ & $52 \pm 4.20$ & 0.02 \\
\hline $\begin{array}{l}\text { Exacerbation requiring } \\
\text { hospitalization in one year }\end{array}$ & $18(26.8 \%)$ & $30(56.6 \%)$ & 0.02 \\
\hline Positive sputum culture & $10(14.9 \%)$ & $36(67.9 \%)$ & 0.03 \\
\hline Antibiotic use & $20(29.8 \%)$ & $30(56.6 \%)$ & 0.03 \\
\hline
\end{tabular}

risk factor for bronchiectasis. ${ }^{5}$ The aim of this study is to identify the factors associated with bronchiectasis, using routine data collected during medical visits from patients diagnosed with moderate or severe COPD.

\section{MATERIALS AND METHODS}

This is cross sectional descriptive study. COPD patients admitted and visited chest specialty clinic from September 2016 to September 2017. Patients diagnosed as COPD included and patient diagnosed of previous bronchiectasis were excluded. Permission was obtained from ethical committee and then the study was conducted. A detailed socio-demographic data of every patient presenting with COPD will be collected either from inpatients and chest clinic records. Information was recorded in structured Performa. The demography included age, sex, smoking history $\&$ history of exposure to indoor pollution by the use of biomass smoke, visit to health care facility with respiratory symptoms in past, history of previous tuberculosis, use of respiratory medications inhalers, domiciliary oxygen and co- morbid conditions. Detailed evaluation of signs and symptoms, chest radiograph, sputum for gram stain and culture and pulmonary function test findings FEV1/FVC, FEV1 values recorded. Duration of hospital stays will be recorded. Those patients subjected to high resolution CT scan and findings recorded in structured Performa.

COPD was defined as a post bronchodilator ratio of FEV1 /FVC, $70 \%$, adjusted for age and height in a patient with a smoking habit of 10 pack-years and $\beta 2$-agonist reversibility on predicted FEV1 of, $15 \%$ and/or $200 \mathrm{~mL}$. COPD was defined as moderate if the postbronchodilator FEV1 was $70 \%$ and severe if the postbronchodilator FEV1 was 50\% and very severe if postbronchodilator FEV1 was 30 percent.
Table 2: Sputum culture positive cases with micro-organism isolated

\begin{tabular}{lcc}
\hline Micro-organism & $\begin{array}{l}\text { COPD without } \\
\text { bronchiectasis } \\
(\mathbf{n}=10)\end{array}$ & $\begin{array}{c}\text { COPD with bron- } \\
\text { chiectasis }\end{array}$ \\
\hline $\begin{array}{l}\text { Pseudomonas } \\
\text { aeruginosa }\end{array}$ & $2(20 \%)$ & $20(55.5 \%)$ \\
\hline $\begin{array}{l}\text { Streptococcus } \\
\text { pneumonia }\end{array}$ & $4(40 \%)$ & $5(13.8 \%)$ \\
\hline Klebsiella pneumonia & $3(30 \%)$ & $6(16.6 \%)$ \\
\hline Haemopilus influenza & $1(10 \%)$ & $2(5.5 \%)$ \\
\hline Citrobacter & 0 & $1(2.7 \%)$ \\
\hline Acinetobacter & 0 & $2(5.5 \%)$ \\
\hline
\end{tabular}

Bronchiectasis was diagnosed clinically and by high resolution computed tomography (HRCT ) scanning. ${ }^{6}$ High resolution $\mathrm{CT}$ was performed on a 16-slice helical CT scanner (bright speed Toshiba) with a pitch 0.98, 200-250 $\mathrm{mA}$ and $120 \mathrm{kV}$. Plain scan of the chest was performed from the level of thoracic inlet to just below the inferior pole of the kidney.

\section{Statistical Analysis:}

SPSS version 15.0 (SPSS; Chicago, Illinois), was used for the statistical analysis. All data were tabulated as mean and SD in the case of quantitative variables and as absolute numbers and percentages in the case of qualitative variables. The Kolmogorov-Smirnov test was used to analyze the distribution of variables. Patients were assigned to two groups, those with and without bronchiectasis. In the bivariate analysis, variables with a normal distribution were analyzed using the Student $t$ test for independent variables or the Mann-Whitney $U$ test in other cases. 


\begin{tabular}{|c|c|}
\hline HRCT findings & $\begin{array}{l}\text { COPD with Bronchiectasis } \\
(\mathrm{n}=53)\end{array}$ \\
\hline \multicolumn{2}{|l|}{ Location } \\
\hline Upper Lobe & $10(18.8 \%)$ \\
\hline Lower Lobe & $43(81.1 \%)$ \\
\hline \multicolumn{2}{|l|}{ Type } \\
\hline Cylindrical & $33(62.2 \%)$ \\
\hline Saccular & $20(37.7 \%)$ \\
\hline \multicolumn{2}{|c|}{ Thickening of bronchial wall } \\
\hline Mild & $20(37.7 \%)$ \\
\hline Moderate & $6(11.3 \%)$ \\
\hline Severe & $8(15 \%)$ \\
\hline Bilateral & $23(43.3 \%)$ \\
\hline Unilateral & $30(56.6 \%)$ \\
\hline \multicolumn{2}{|l|}{ Other findings } \\
\hline Consolidation (fig.1B) & $10(18.86 \%)$ \\
\hline Atelectasis & $6(11.3 \%)$ \\
\hline Adenopathy & $8(15.0 \%)$ \\
\hline Fibrosis (fig.1A) & $10(18.8 \%)$ \\
\hline
\end{tabular}

\section{RESULTS}

Total 120 patients of COPD were analyzed among them 67 patients $(55.8 \%)$ were COPD without bronchiectasis while 53 patients $(44.1 \%)$ had COPD with bronchiectasis. Forty patients $(75.4 \%)$ were male and 13 patients $(24.5 \%)$ were female in COPD with bronchiectasis. Most of the patient COPD with bronchiectasis had moderate to severe airflow limitation.(Table 1).

Thirty patients (56.6\%) having COPD with bronchiectasis and 18 patients (26.8\%) having COPD without bronchiectasis had exacerbation in one year requiring hospitalisation. (Table 1) Thirty-six patients (67.9\%) had positive sputum culture among patients with COPD with bronchiectasis and 10 patients $(14.9 \%)$ had positive sputum culture in COPD without bronchiectasis. Most common micro-organisms isolated were Pseudomonas Aeruginosa ( $n=20 ; 55.5 \%)$ followed by Klebsiellae Pneumoniae (n-6; 16.6\%) among COPD with bronchiectasis group. (Table 2)

Among COPD with bronchiectasis patient who had undergone high resolution CT scan chest (fig. 1A\&B), 43 patients (81.1\%) had lower lobe predominance involvement. Bilateral involvement was detected in $30(56.6 \%)$ patients. (Table 3)

\section{DISCUSSION}

The present study examined a well characterized group of hospital outpatients and inpatients with moderate to severe COPD. HRCT scans of the chest were performed on patients in the stable state and the extent of bronchiectasis was quantified. HRCT is now accepted as the imaging modality of choice for the evaluation of bronchiectasis.

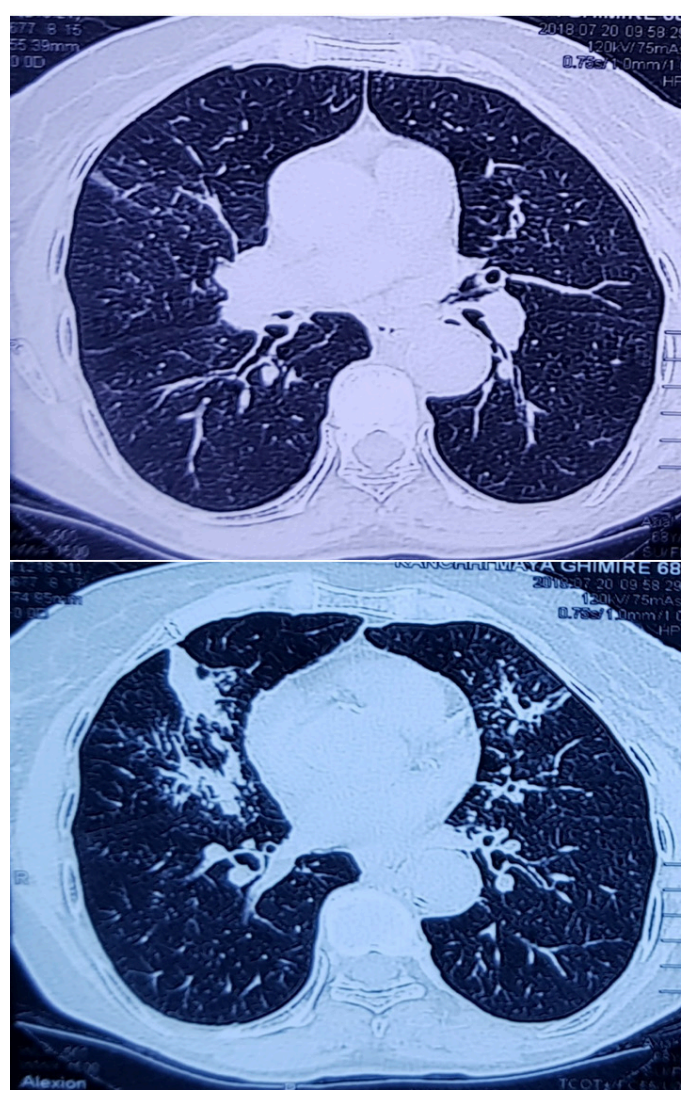

Figure 1A: Cylindrical broncheictasis with fibrosis B.Cylindrical broncheictasis with consolidation

Prevalence of bronchiectasis in patient with moderate to severe COPD was observed in 53 patients(44.1\%) and it was primarily of cylindrical type(62.2\%), having lower lobe predominance $43(81.1 \%)$ with similar findings by Patel et al and Martinez $\mathrm{M}$ et al. $57.6 \%$ in moderate COPD. ${ }^{7,8}$ Other recent studies in patients with COPD with all GOLD stages of disease showed bronchiectasis rate of $27 \%$ in ECLIPSE study. ${ }^{9}$

The second factor associated with presence of bronchiectasis was positive sputum culture. Thirty six $(67.9 \%)$ patients of COPD with bronchiectasis had positive sputum culture. The result is similar to study done by Patel etal. ${ }^{6} \mathrm{He}$ observed that, in 54 patients with moderate to severe COPD, that greater bronchial colonization by potentially pathogenic microorganism (PPM) was associated with the presence of bronchiectasis According to the hypotheses of Cole et $\mathrm{al},{ }^{10}$ it is possible that the presence of PPM and concomitant proteolytic products is responsible for triggering the mechanism that generates bronchiectasis, along with the chronic inflammation of the bronchial mucosa secondary to this phenomenon in some patients with COPD.

Patel et $\mathrm{al}^{6}$ observed that even though the number of exacerbations was not related to bronchiectasis in their study, patients with bronchiectasis did experience longer exacerbations. Our results indicate that the presence of $p$. aeruginosa could potentially be a marker for bronchiectasis 
in patients with moderate to severe COPD, similar results was found by Martinez-Garcia et al. ${ }^{11}$ The rate of exacerbation requiring hospitalisation in previous year was $56.6 \%$ among patient with bronchiectasis with COPD in our study. When bronchiectasis is detected in patient who present with severe functional COPD their prognosis is poorer. Results from patients with moderate to severe COPD, the presence of severe airflow obstruction, the isolation of a PPM in a sputum sample, and the need for at least one hospital admission in the previous year are associated with bronchiectasis. When bronchiectasis is detected in patients who present more severe functional COPD8 with PPMs in the bronchial mucosa and exacerbations particularly serious ones $^{12}$, their prognosis is poorer; this underlines the need for the early identification of the specific phenotype of patients with COPD and bronchiectasis, although further studies are needed to evaluate whether the presence of bronchiectasis worsens the prognosis of patients with COPD.

\section{CONCLUSION}

Moderate to severe COPD patient had radiologic prevalance of bronchiectasis resulting in severe airflow limitation, with frequent exacerbation and colonisation with isolation of organisms requiring frequent hospitalisation. This finding will help to identify factors associated with bronchiectasis and to initiate appropriate therapy.

\section{Conflict of interest: None}

\section{REFERENCES}

1. StockleyRA. Neutrophils and the pathogenesis of COPD. Chest 2002;121:151S-5S. Crossref

2. FuschilloS, De Felice A, Balzano G. Mucosal inflammation in idiopathic bronchiectasis: cellular and molecular mechanisms .EurRespirJ. 2008;31:396-406. $\underline{\text { Crossref }}$
3. National Collaborating Center for Chronic Conditions. Chronic obstructive pulmonary disease. National clinical guideline on management of chronic obstructive pulmonary diseasein adults in primary and secondary care. Thorax 2004;59:1-232.

4. Martínez-GarcíaMA ,Soler-Catalu-a JJ, Perpi-á-ordera M ,RománSánchez P, Soriano J . Factors associated with lungfunction decline in adult patients with stable non-cystic fibrosisbronchiectasis. Chest 2007;132:1565-72. Crossref

5. I.S. Patel, I. Vlahos, T.M.A. Wilkinson, et al, Bronchiectasis, exacerbation indices, and inflammation in chronic obstructive pulmonary disease, Am. J. Respir. Crit. Care Med. 2004;170:400-7. Crossref

6. Smith IE, Flower CD. Reviw article:imaging in bronchiectasis. Br J Radiol 1996;69:589-93. Crossref

7. Patel IS, Vlahos I, Wilkinson TMA et al. Bronchiectasis, exacerbation indices, and inflammation in chronic obstructive pulmonary disease .Am J RespirCrit Care Med 2004;170:400-7. Crossref

8. M.A. Martinez-Garcia, J.J. Soler-Cataluria, Y.D. Sanz, et al,Factors associated with bronchiectasis in patients with COPD, Chest 2011;140:1130-7. Crossref

9. A. Agusti, P.M. Calverley, B. Celli, et al, Evaluation of COPD longitudinally to identify predictive surrogate endpoints (ECLIPSE) cohort, Respir. Res.2010;11:122. Crossref

10. Cole PJ. Inflammation: a two-edged sword-the model of bronchiectasis. Eur J Respir Dis Suppl 1986;147:6-15. Crossref

11. Soler-Catalu-a JJ ,Martínez-García MA, Román Sánchez P, Salcedo E, Navarro M, Ochando R. Severe acute exacerbations and mortality in patients with chronic obstructive pulmonary disease.Thorax 2005;60:925-31. $\underline{\text { Crossref }}$

12. Celli BR, Cote CG, Marin JM, et al. The body-mass index, airflow obstruction, dyspnea, and exercise capacity index in chronic obstructive pulmonary disease. N Engl J Med 2004; 350:1005-12. Crossref 